\title{
Body Area Networks: Radio Channel Modelling and Propagation Characteristics
}

\author{
Aastha Gupta, Thushara D. Abhayapala \\ Department of Information Engineering \\ Research School of Information Sciences and Engineering \\ Australian National University \\ Canberra, ACT 0200 \\ Australia \\ aastha.gupta@rsise.anu.edu.au, thushara.abhayapala@anu.edu.au
}

\begin{abstract}
Human body will act as a transmission channel in wearable wireless devices in the near future. Inclusion of the body as a transmission channel will see future wireless networks rely heavily on Body Area Networks (BAN) with applications in medical and personal area networks. In order to build BAN devices, it is imperative to model the channel accurately. Channel measurements are important, however, a closer look on the body channel can only be attained through Electromagnetic (EM) propagation modelling. This paper presents a preliminary analytical EM channel model for BAN. Specifically, the dyadic Green's function for a simple cylindrical human body model is used to propose a channel model. Four possible cases are considered, where the transmitter and receiver are either inside or outside of the body. An exact analytical expression is derived for the case where both the transmitter and receiver are outside of the body. This case is then used to show how the received signal power varies around the body, with the receiver at a constant radial distance from the cylindrical axis of the body.
\end{abstract}

\section{INTRODUCTION}

Medicine is an area of technology that has often been ignored by the Telecommunications community. As the aged population is increasing and healthcare professionals are decreasing, hospitals throughout the world are facing a unique problem. There is an arising need to remotely monitor and treat patients. To realize this task, it is required to communicate between portable sensor devices worn on and implanted within the body of the user and an external interface. This will be achieved by Body Area Networks (BAN).

BAN can be used not only on remote patients but also enables to make patients wireless within the hospital, especially, in intensive care units and operating theatres. Not only this would enhance patient comfort but also it would make the work of doctors and nurses a lot more efficient and easy. On site accident communication can be performed via BAN, wherein a paramedic strap on the BAN sensors which can start providing vital information to the hospital directly, hence increasing efficiency, reducing reaction time, and saving crucial life critical minutes. There have been a few projects throughout the world which have started working in Healthcare BAN and related devices such as Human++ [1], Memswear [2], Motes [3], and Ambulant monitoring [4]. As BAN deals with connecting the body to wireless devices, it has applications in numerous other areas as well; such as, sports, entertainment, and defence forces.

To build any wireless device, the first essential step is to study the transmission channel and model it accurately. In this attempt, a few research groups throughout the world have initiated channel modelling [5]. They have performed measurement campaigns and path loss studies for wireless nodes on the body [6], [7], [8], [9], [10], [11], [12]. Some have also considered implanted devices, an area of BAN called Intra-body Communication [13]. Due to the short-range low data-rate communication in BAN scenarios, measurement groups have considered UWB as the appropriate air-interface. Although there are quite a few measurement campaigns, each model developed by them is only a path loss model and does not provide any detail description of the propagation channel.

In order to develop a general and accurate BAN channel model, it is important to study the propagation mechanism of wireless radio waves on and inside the body. Such a study will reveal the underlying propagation characteristics. This will assist in the development of enhanced BAN transceivers, which are more suited to the body environment. The human body is a very complex environment and has not been studied explicitly for wireless communication. Although, a while ago the human body was under focus for the measurement of electromagnetic absorption studies, such as specific absorption rate and dosimetry [14], [15], [16], [17].

For a BAN channel model, it is required to determine the electromagnetic field at each point on/inside the body for a given position of the transmitter on/inside the body. This is a huge problem numerically, which requires enormous amounts of computational power. Therefore, it is desirable to derive an analytical expression which performs this objective. Analytically resolving this problem means solving the Maxwell's equations for each point of the body. In effect this determines which propagation mechanism is taking place, i.e. diffraction, reflection, transmission, surface waves [18]. An elegant manner of doing this task is using the dyadic Green's function.

Dyadic Green's functions have been used in Electromagnetic (EM) theory and have solutions for canonical problems, 
such as cylinders, multilayered cylinders and spheres [19], [20], [21].

In this paper, as a first step to understand the propagation characteristics for the body, we present an analytical channel model using EM theory for a simple body structure. Section II provides an overview of the BAN channel along with a brief description of the problem formulation. Detailed expressions of dyadic Greens functions are provided in Section III, followed by a special case study in Section IV. Finally, simulation results are presented in Section $\mathrm{V}$ to illustrate the analytical model and provide a view as to how the electric field varies on the human body.

\section{BAN CHANNEL}

\section{A. Human Model}

For this study, a typical human body is assumed to be a cylinder with radius $a$ filled with a homogeneous dielectric material of permittivity $\epsilon_{1}$ and permeability $\mu_{1}$. In future work, we expect to develop a more realistic human model. However, the work presented in this paper would act as a building block to construct a more complicated and accurate human model.

We use the cylindrical coordinate system to represent points in and around the body where the $z$-axis is aligned with the axis of the cylindrical body. A point in space is denoted by $\boldsymbol{x}$ which represents $(r, \phi, z)$ coordinates in the cylindrical coordinate system. We denote $\widehat{\boldsymbol{r}}, \widehat{\phi}$, and $\widehat{z}$ as unit vectors along radial, azimuth, and ' $z$ ' directions.

\section{B. Electric Field}

Let $\boldsymbol{x}_{0}$ be the location of the transmitting antenna and $\boldsymbol{x}$ be the location of the receiver. These two points could be inside $^{1}$, outside, or on the body. Let $\boldsymbol{E}(\boldsymbol{x})$ be the electric field at point $\boldsymbol{x}$ due to a current source $\boldsymbol{J}\left(\boldsymbol{x}_{0}\right)$ located at $\boldsymbol{x}_{0}$. Both the current source and the resulting electric field have three components in directions $\widehat{\boldsymbol{r}}, \widehat{\phi}$, and $\widehat{\boldsymbol{z}}$.

The general formulation for the electric field $\boldsymbol{E}(\boldsymbol{x})$ excited by an electric current source $\boldsymbol{J}\left(\boldsymbol{x}_{0}\right)$ can be written as

$$
\boldsymbol{E}(\boldsymbol{x})=i \omega \mu_{p} \iiint_{V} \overline{\bar{G}}\left(\boldsymbol{x}, \boldsymbol{x}_{0}\right) \boldsymbol{J}\left(\boldsymbol{x}_{0}\right) d V
$$

where $V$ is the volume occupied by the sources, $\mu_{p}$ is the magnetic permeability of the medium where the receiver is located, $\omega$ is the radian frequency of transmission and $\overline{\bar{G}}(\cdot, \cdot)$ stands for the dyadic Green's function. The concept of dyadic Green's functions is well known amongst the Antennas and Propagation community. However, for the benefit of the readers, we provide a brief description of the dyadic Green's function and some of the associated algebra of dyads.

\section{Dyadic Green's function}

The Dyadic Green's function consists of nine components, corresponding to the dyads: $\widehat{r} \widehat{r}, \widehat{r} \widehat{\phi}, \widehat{r} \widehat{z}, \widehat{\phi} \widehat{r}, \widehat{\phi} \widehat{\phi}, \widehat{\phi} \widehat{z}, \widehat{z} \widehat{r}, \widehat{z} \widehat{\phi}$, and $\widehat{z} \widehat{z}$. Usually, a $3 \times 3$ matrix is used to represent these nine components.

\footnotetext{
${ }^{1} \mathrm{~A}$ transmitter inside the body is applicable in a case of implanted devices.
}

The dyadic Green's function can be viewed as the transfer function between the source, and the measuring point of the resulting electric field. The properties of the transmission medium, reflection, and transmission coefficients between different media are all part of the dyadic Green's function. Since the interface between two mediums is close to both the transmitter and receiver, none of the farfield assumptions used in wireless channel modelling are valid in this case. Due to the reflection, scattering, and transmission effects, a source pointing in one direction may produce electric field in the other two orthogonal directions as well. For example, in the dyadic Green's function, the component in $\widehat{r} \widehat{\phi}$ can be described as the resulting electric field component in the $\widehat{r}$ direction due to a unit current source in the $\widehat{\phi}$ direction.

\section{Problem Formulation}

In this paper, we use existing electro-magnetic (EM) theory to write an appropriate expression for the dyadic Green's function of the approximate human body model described in Section II-A. We also use the developed model to characterize the receiver power level as a function of the receiver position.

\section{General Expression of Dyadic Green's FUNCTION}

Due to the cylindrical symmetry of the human body model, we use cylindrical vector eigen functions ${ }^{2}$ to write the dyadic Green's function.

\section{A. Cylindrical Vector Eigen Functions}

The cylindrical vector eigen functions are the building blocks of various dyadic Green's functions [22]. We denote them by $\boldsymbol{L}_{n h k}(\boldsymbol{x}), \boldsymbol{M}_{n h k}(\boldsymbol{x}), \boldsymbol{N}_{n h k}(\boldsymbol{x})$ where $n$ is an integer, $h$ is a real number, $k$ is the wave number of the medium, and $\boldsymbol{x}$ is a point in space. All of them are solutions to the vector Helmholtz equation and have three components in $\widehat{\boldsymbol{r}}, \widehat{\phi}$ and $\widehat{z}$. The vector eigen functions are given by [22]

$$
\begin{aligned}
\boldsymbol{L}_{n h k}(\boldsymbol{x}) & =\nabla \psi_{n h k}(\boldsymbol{x}) \\
\boldsymbol{M}_{n h k}(\boldsymbol{x}) & =\nabla \times\left[\psi_{n h k}(\boldsymbol{x}) \widehat{\boldsymbol{z}}\right] \\
\boldsymbol{N}_{n h k}(\boldsymbol{x}) & =\frac{1}{k} \nabla \times \nabla \times\left[\psi_{n h k}(\boldsymbol{x}) \widehat{\boldsymbol{z}}\right]
\end{aligned}
$$

where $\boldsymbol{x} \equiv(r, \phi, z)$ represents a point in space, $\nabla$ is the Laplacian operator in the cylindrical coordinate system, and $\psi_{n h k}(\boldsymbol{x})$ is the scalar eigen function which is a solution of the scalar Helmholtz equation. The scalar eigen function is given by [22]

$$
\psi_{n h k}(\boldsymbol{x})=Z_{n}(\eta r) e^{j n \phi} e^{j n z}
$$

where $Z_{n}(\cdot)$ is a general cylindrical function of order $n, \eta$ is the propagation constant in $\widehat{r}$ direction with $k^{2}=\eta^{2}+h^{2}$. For the case of the body channel considered in this paper, only two types of cylindrical functions exit namely the Bessel functions $J_{n}(\cdot)$ and the Hankel function of first kind $H_{n}^{(1)}(\cdot)$.

\footnotetext{
${ }^{2}$ In some literature, these are also referred to as cylindrical vector wave functions.
} 
These two functions physically represent the standing waves and outgoing waves respectively.

The vector eigen functions in (2), (3), (4) can be written as [22]

$$
\begin{aligned}
\boldsymbol{L}_{n h k}(\boldsymbol{x}) & =\left(\begin{array}{c}
\frac{d Z_{n}(\eta r)}{d r} e^{j n \phi} e^{j h z} \\
\frac{n Z_{n}(\eta r)}{r} e^{-j n \phi} e^{j h z} \\
j h Z_{n}(\eta r) e^{j n \phi} e^{j h z}
\end{array}\right), \\
\boldsymbol{M}_{n h k}(\boldsymbol{x}) & =\left(\begin{array}{c}
\frac{n Z_{n}(\eta r)}{r(\eta r} e^{-j n \phi} e^{j h z} \\
\frac{-d \eta r)}{d r} e^{j n \phi} e^{j h z} \\
0
\end{array}\right),
\end{aligned}
$$

and

$$
\boldsymbol{N}_{n h k}(\boldsymbol{x})=\left(\begin{array}{c}
\frac{i h}{k} \frac{d Z_{n}(\eta r)}{d r} e^{j n \phi} e^{j h z} \\
\frac{i h n}{r k} Z_{n}(\eta r) e^{-j n \phi} e^{j h z} \\
\frac{\eta^{2}}{k} Z_{n}(\eta r) e^{j n \phi} e^{j h z}
\end{array}\right) .
$$

Note that the vector eigen functions are orthogonal among themselves as well as with respect to each other [19].

\section{B. Scattering Superposition}

Using the principle of scattering superposition [19], we can write the dyadic Green's function (DGF) as the superposition of the direct DGF $\overline{\bar{G}}_{d}\left(\boldsymbol{x}, \boldsymbol{x}_{0}\right)$ and the scattering DGF $\overline{\bar{G}}_{s}\left(\boldsymbol{x}, \boldsymbol{x}_{0}\right)$. The direct DGF $\overline{\bar{G}}_{d}\left(\boldsymbol{x}, \boldsymbol{x}_{0}\right)$ corresponds to the contribution of the direct waves from the source to the measuring point. Whereas, the scattering DGF $\overline{\bar{G}}_{s}\left(\boldsymbol{x}, \boldsymbol{x}_{0}\right)$ is from the contribution of the reflection and transmission waves due to the presence of the dielectric interface. Thus,

$$
\overline{\bar{G}}\left(\boldsymbol{x}, \boldsymbol{x}_{0}\right)=\overline{\bar{G}}_{d}\left(\boldsymbol{x}, \boldsymbol{x}_{0}\right)+\overline{\bar{G}}_{s}\left(\boldsymbol{x}, \boldsymbol{x}_{0}\right) .
$$

\section{Direct Component of DGF}

The direct component of DGF is given by [19]

$$
\begin{aligned}
& \overline{\bar{G}}_{d}\left(\boldsymbol{x}, \boldsymbol{x}_{0}\right)=\frac{\widehat{r} \widehat{r}}{k^{2}} \delta\left(\boldsymbol{x}-\boldsymbol{x}_{0}\right)+\frac{j}{8 \pi} \int_{-\infty}^{\infty} d h \sum_{n=-\infty}^{\infty} \frac{1}{\eta^{2}} \\
& \times\left\{\begin{array}{l}
\boldsymbol{M}_{n h k}^{(1)}(\boldsymbol{x}) \otimes \boldsymbol{M}_{n h k}^{*}\left(\boldsymbol{x}_{0}\right)+\boldsymbol{N}_{n h k}^{(1)}(\boldsymbol{x}) \otimes \boldsymbol{N}_{n h k}^{*}\left(\boldsymbol{x}_{0}\right) \\
\boldsymbol{M}_{n h k}(\boldsymbol{x}) \otimes \boldsymbol{M}_{n h k}^{(1)^{*}}\left(\boldsymbol{x}_{0}\right)+\boldsymbol{N}_{n h k}(\boldsymbol{x}) \otimes \boldsymbol{N}_{n h k}^{(1)^{*}}\left(\boldsymbol{x}_{0}\right)
\end{array}\right.
\end{aligned}
$$

where the first case is for $r>r_{0}$ and the second case is for $r<r_{0}$. We use $\otimes$ to denote 'dyadic product' and.$^{*}$ for conjugation. $H_{n}^{(1)}$ should be chosen for $Z_{n}$ if vector eigen function has the superscript (1) (outgoing waves), otherwise $J_{n}$ should be used (standing waves).

\section{Scattering Component of DGF}

We can write the scattering component of the DGF for four different scenarios:- (i) Both the transmitter and receiver are inside the body: $\overline{\bar{G}}_{s}^{(11)}\left(\boldsymbol{x}, \boldsymbol{x}_{0}\right)$; (ii) The transmitter is located inside the body and the receiver is located outside: $\overline{\bar{G}}_{s}^{(12)}\left(\boldsymbol{x}, \boldsymbol{x}_{0}\right)$; (iii) Both the transmitter and receiver are located outside the body: $\overline{\bar{G}}_{s}^{(22)}\left(\boldsymbol{x}, \boldsymbol{x}_{0}\right)$; and (iv) The transmitter is located outside the body and the receiver is located inside: $\overline{\bar{G}}_{s}^{(21)}\left(\boldsymbol{x}, \boldsymbol{x}_{0}\right)$. In the above notation of scattering DGF, the two numbers in the superscript represent the medium where the receiver and transmitter are located in that order, ' 1 ' represents the medium inside the body and, ' 2 ' represents the free space (medium outside the body). Below, we write the appropriate DGF for each case.

1) Transmitter and receiver located inside the body: In this case, the DGF can be written as

$$
\begin{aligned}
& \overline{\bar{G}}_{s}^{(11)}\left(\boldsymbol{x}, \boldsymbol{x}_{0}\right)=\int_{-\infty}^{\infty} d h \sum_{n=-\infty}^{\infty} \frac{j}{8 \pi \eta_{1}^{2}} \times \\
& {\left[\begin{array}{ll}
\boldsymbol{N}_{n h k_{1}}(\boldsymbol{x}) & \left.\boldsymbol{M}_{n h k_{1}}(\boldsymbol{x})\right] \boldsymbol{R}_{12}\left[\begin{array}{l}
\boldsymbol{N}_{n h k_{1}}^{*}\left(\boldsymbol{x}_{0}\right)^{T} \\
\boldsymbol{M}_{n h k_{1}}^{*}\left(\boldsymbol{x}_{0}\right)^{T}
\end{array}\right]
\end{array}\right.}
\end{aligned}
$$

where $\boldsymbol{R}_{12}$ contains the reflection coefficients which have been derived in the literature using boundary conditions. The reflection coefficient matrix is given by [19]

$$
\begin{aligned}
\boldsymbol{R}_{12} & =\left[\boldsymbol{J}_{n}\left(\eta_{1} a\right) H_{n}^{(1)}\left(\eta_{2} a\right)-\boldsymbol{H}_{n}\left(\eta_{2} a\right) J_{n}\left(\eta_{1} a\right)\right]^{-1} \\
& \times\left[\boldsymbol{H}_{n}\left(\eta_{2} a\right) H_{n}^{(1)}\left(\eta_{1} a\right)-\boldsymbol{H}_{n}\left(\eta_{1} a\right) J_{n}\left(\eta_{2} a\right)\right]
\end{aligned}
$$

where $a$ is the radius of the cylindrical body model, $\eta_{1}^{2}=$ $k_{1}^{2}-h^{2}, \eta_{2}^{2}=k_{2}^{2}-h^{2}, k_{1}^{2}=\omega^{2} \mu_{1} \epsilon_{1}, k_{2}^{2}=\omega^{2} \mu_{2} \epsilon_{2}$, subscript ' 1 ' represents the medium inside the body and subscript ' 2 ' represents the medium outside the body. The $2 \times 2$ matrices $\boldsymbol{J}_{n}(\eta a)$ and $\boldsymbol{H}_{n}(\eta a)$ are defined as

$$
\begin{aligned}
& \boldsymbol{B}_{n}\left(\eta_{p} a\right)=\frac{1}{\eta_{p}^{2} a} \\
& {\left[\begin{array}{cc}
j \omega \epsilon_{p} \eta_{p} a \dot{B}_{n}\left(\eta_{p} a\right) & -n h B_{n}\left(\eta_{p} a\right) \\
-n h B_{n}\left(\eta_{p} a\right) & -j \omega \mu_{p} \eta_{p} a \dot{B}_{n}\left(\eta_{p} a\right)
\end{array}\right]}
\end{aligned}
$$

where $B_{n}$ is either $H_{n}^{(1)}$ or $J_{n}, \dot{B}(\cdot)$ represents the derivative of $B$ with respect to the whole argument, and $p=1,2$.

2) Transmitter located inside and receiver located outside the body: In this case, the DGF can be written as [19]

$$
\begin{aligned}
& \overline{\bar{G}}_{s}^{(21)}\left(\boldsymbol{x}, \boldsymbol{x}_{0}\right)=\int_{-\infty}^{\infty} d h \sum_{n=-\infty}^{\infty} \frac{j}{8 \pi \eta_{2}^{2}} \times \\
& {\left[\begin{array}{ll}
\boldsymbol{N}_{n h k_{2}}^{(1)}(\boldsymbol{x}) & \boldsymbol{M}_{n h k_{2}}^{(1)}(\boldsymbol{x})
\end{array}\right] \boldsymbol{T}_{12}\left[\begin{array}{l}
\boldsymbol{N}_{n h k_{1}}^{*}\left(\boldsymbol{x}_{0}\right)^{T} \\
\boldsymbol{M}_{n h k_{1}}^{*}\left(\boldsymbol{x}_{0}\right)^{T}
\end{array}\right]}
\end{aligned}
$$

where $\boldsymbol{T}_{12}$ is the transmission coefficient matrix given by

$$
\begin{aligned}
\boldsymbol{T}_{12} & =\frac{2 \omega}{\pi \eta_{1}^{2} a}\left[\boldsymbol{J}_{n}\left(\eta_{1} a\right) H_{n}^{(1)}\left(\eta_{2} a\right)-\boldsymbol{H}_{n}\left(\eta_{2} a\right) J_{n}\left(\eta_{1} a\right)\right]^{-1} \\
& \times\left(\begin{array}{cc}
\epsilon_{1} & 0 \\
0 & -\mu_{1}
\end{array}\right) .
\end{aligned}
$$

3) Both transmitter and receiver located outside the body: In this case, the DGF can be written as

$$
\begin{aligned}
& \overline{\bar{G}}_{s}^{(22)}\left(\boldsymbol{x}, \boldsymbol{x}_{0}\right)=\int_{-\infty}^{\infty} d h \sum_{n=-\infty}^{\infty} \frac{j}{8 \pi \eta_{2}^{2}} \times \\
& {\left[\begin{array}{ll}
\boldsymbol{N}_{n h k_{2}}^{(1)}(\boldsymbol{x}) & \boldsymbol{M}_{n h k_{2}}^{(1)}(\boldsymbol{x})
\end{array}\right] \boldsymbol{R}_{21}\left[\begin{array}{l}
\boldsymbol{N}_{n h k_{2}}^{(1)^{*}}\left(\boldsymbol{x}_{0}\right)^{T} \\
\boldsymbol{M}_{n h k_{2}}^{(1)^{*}}\left(\boldsymbol{x}_{0}\right)^{T}
\end{array}\right]}
\end{aligned}
$$

where $\boldsymbol{R}_{21}$ is the reflection coefficient matrix given by

$$
\begin{aligned}
\boldsymbol{R}_{21} & =\left[\boldsymbol{J}_{n}\left(\eta_{1} a\right) H_{n}^{(1)}\left(\eta_{2} a\right)-\boldsymbol{H}_{n}\left(\eta_{2} a\right) J_{n}\left(\eta_{1} a\right)\right]^{-1} \\
& \times\left[\boldsymbol{J}_{n}\left(\eta_{2} a\right) J_{n}\left(\eta_{1} a\right)-\boldsymbol{J}_{n}\left(\eta_{1} a\right) J_{n}\left(\eta_{2} a\right)\right] .
\end{aligned}
$$


4) Transmitter located outside and receiver inside the body: In this case, the DGF can be written as

$$
\begin{aligned}
& \overline{\bar{G}}_{s}^{(12)}\left(\boldsymbol{x}, \boldsymbol{x}_{0}\right)=\int_{-\infty}^{\infty} d h \sum_{n=-\infty}^{\infty} \frac{j}{8 \pi \eta_{1}^{2}} \times \\
& {\left[\begin{array}{ll}
\boldsymbol{N}_{n h k_{1}}(\boldsymbol{x}) & \boldsymbol{M}_{n h k_{1}}(\boldsymbol{x})
\end{array}\right] \boldsymbol{T}_{21}\left[\begin{array}{c}
\boldsymbol{N}_{n h k_{2}}^{(1)^{*}}\left(\boldsymbol{x}_{0}\right)^{T} \\
\boldsymbol{M}_{n h k_{2}}^{(1)^{*}}\left(\boldsymbol{x}_{0}\right)^{T}
\end{array}\right]}
\end{aligned}
$$

where $\boldsymbol{T}_{12}$ is the transmission coefficient matrix and given by

$$
\begin{aligned}
\boldsymbol{T}_{21} & =\frac{2 \omega}{\pi \eta_{2}^{2} a}\left[\boldsymbol{J}_{n}\left(\eta_{1} a\right) H_{n}^{(1)}\left(\eta_{2} a\right)-\boldsymbol{H}_{n}\left(\eta_{2} a\right) J_{n}\left(\eta_{1} a\right)\right]^{-1} \\
& \times\left(\begin{array}{cc}
\epsilon_{2} & 0 \\
0 & -\mu_{2}
\end{array}\right) .
\end{aligned}
$$

\section{Special Case: Transmitter and Receiver} LOCATED OUTSIDE OF THE BODY

In this section, we write (16) in an explicit form which can be used to study the propagation characteristics around the human body (model) by simulation.

Let us write (16) as

$$
\overline{\bar{G}}_{s}^{(22)}\left(\boldsymbol{x}, \boldsymbol{x}_{0}\right)=\int_{-\infty}^{\infty} \sum_{n=-\infty}^{\infty} \frac{j}{8 \pi \eta_{2}^{2}} \overline{\bar{G}}_{n h}^{(22)}\left(\boldsymbol{x}, \boldsymbol{x}_{0}\right) d h
$$

where $\overline{\bar{G}}_{n h}^{(22)}\left(\boldsymbol{x}, \boldsymbol{x}_{0}\right)$ is defined as

$$
\begin{aligned}
& \overline{\bar{G}}_{n h}^{(22)}\left(\boldsymbol{x}, \boldsymbol{x}_{0}\right)= \\
& {\left[\begin{array}{ll}
\boldsymbol{N}_{n h k_{2}}^{(1)}(\boldsymbol{x}) & \boldsymbol{M}_{n h k_{2}}^{(1)}(\boldsymbol{x})
\end{array}\right] \boldsymbol{R}_{21}\left[\begin{array}{c}
\boldsymbol{N}_{n h k_{2}}^{(1)^{*}}\left(\boldsymbol{x}_{0}\right)^{T} \\
\boldsymbol{M}_{n h k_{2}}^{(1)^{*}}\left(\boldsymbol{x}_{0}\right)^{T}
\end{array}\right] .}
\end{aligned}
$$

In the matrix form, the dyadic $\overline{\bar{G}}_{n h}^{(22)}\left(\boldsymbol{x}, \boldsymbol{x}_{0}\right)$ is a $3 \times 3$ matrix and we denote the $(p, q)$ element as $g_{p q}$. We have derived a closed form expression for $g_{p q}$ (proof will be provided in a future publication)

$$
g_{p q}=r_{11} n_{p} n_{q}^{o}+r_{21} m_{p} n_{q}^{o}+r_{12} n_{p} m_{q}^{o}+r_{22} m_{p} m_{q}^{o}
$$

where $r_{11}, r_{21}, r_{12}$, and $r_{22}$ are the 4 elements of $\boldsymbol{R}_{21}$, and $\left[n_{1}, n_{2}, n_{3}\right]^{T}=\boldsymbol{N}_{n h k_{2}}^{(1)}(\boldsymbol{x}), \quad\left[n_{1}^{o}, n_{2}^{o}, n_{3}^{o}\right]=$ $\boldsymbol{N}_{n h k_{2}}^{(1)^{*}}\left(\boldsymbol{x}_{0}\right),\left[m_{1}, m_{2}, m_{3}\right]=\boldsymbol{M}_{n h k_{2}}^{(1)}(\boldsymbol{x})$, and $\left[m_{1}^{o}, m_{2}^{o}, m_{3}^{o}\right]=$ $\boldsymbol{M}_{n h k_{2}}^{(1)^{*}}\left(\boldsymbol{x}_{0}\right)$. We also derived explicit expressions for transmission coefficients as

$$
\begin{aligned}
& r_{11}=\frac{c_{2} e_{1}-d_{1} d_{2}}{c_{1} e_{1}-d_{1}^{2}} \\
& r_{12}=\frac{d_{2} e_{1}-d_{1} e_{2}}{c_{1} e_{1}-d_{1}^{2}} \\
& r_{21}=\frac{c_{1} d_{2}-c_{2} d_{1}}{c_{1} e_{1}-d_{1}^{2}} \\
& r_{22}=\frac{c_{1} e_{2}-d_{1} d_{2}}{c_{1} e_{1}-d_{1}^{2}}
\end{aligned}
$$

where

$$
\begin{aligned}
c_{1}= & j \omega\left[\frac{\epsilon_{1}}{\eta_{1}} \dot{J}_{n}\left(\eta_{1} a\right) H_{n}^{(1)}\left(\eta_{2} a\right)\right. \\
& \left.-\frac{\epsilon_{2}}{\eta_{2}} \dot{H}_{n}^{(1)}\left(\eta_{2} a\right) J_{n}\left(\eta_{1} a\right)\right],
\end{aligned}
$$

$$
\begin{gathered}
c_{2}=j \omega\left[\frac{\epsilon_{2}}{\eta_{2}} \dot{J}_{n}\left(\eta_{2} a\right) J_{n}\left(\eta_{1} a\right)\right. \\
\left.-\frac{\epsilon_{1}}{\eta_{1}} \dot{J}_{n}\left(\eta_{1} a\right) J_{n}\left(\eta_{2} a\right)\right], \\
d_{1}=\frac{n h}{a}\left(\frac{1}{\eta_{2}^{2}}-\frac{1}{\eta_{1}^{2}}\right) J_{n}\left(\eta_{1} a\right) H_{n}^{(1)}\left(\eta_{2} a\right), \\
d_{2}=\frac{n h}{a}\left(\frac{1}{\eta_{2}^{2}}-\frac{1}{\eta_{1}^{2}}\right) J_{n}\left(\eta_{1} a\right) J_{n}\left(\eta_{2} a\right), \\
e_{1}=-j \omega\left[\frac{\mu_{1}}{\eta_{1}} \dot{J}_{n}\left(\eta_{1} a\right) H_{n}^{(1)}\left(\eta_{2} a\right)\right. \\
\left.-\frac{\mu_{2}}{\eta_{2}} \dot{H}_{n}^{(1)}\left(\eta_{2} a\right) J_{n}\left(\eta_{1} a\right)\right],
\end{gathered}
$$

and

$$
\begin{aligned}
e_{2}= & -j \omega\left(\frac{\mu_{2}}{\eta_{2}} \dot{J}_{n}\left(\eta_{2} a\right) J_{n}\left(\eta_{1} a\right)\right. \\
& \left.-\frac{\mu_{1}}{\eta_{1}} \dot{J}_{n}\left(\eta_{1} a\right) J_{n}\left(\eta_{2} a\right)\right) .
\end{aligned}
$$

In the next section, we use (20) to simulate a radio propagation environment around our simple human body model when both the transmitter and receiver are located outside the body.

\section{Simulation}

\section{A. Body parameters}

We simulate the radio propagation environment for the cylindrical human body model model develop in this paper, having a radius $a=15 \mathrm{~cm}$, electric permittivity $\epsilon_{2}=2.563 \times$ $10^{-10}$ and magnetic permeability $\mu_{2}=1.256 \times 10^{-6}$ (the human body is assumed to have a permeability approximately equal to air, See: http://niremf.ifac.cnr.it/tissprop/). The dielectric constants used for the human body are a mean value of all tissues and composite body material. The surrounding homogenous medium is assumed to be air with dielectric constants, $\epsilon_{1}=8.8542 \times 10^{-12}$ and $\mu_{1}=1.256 \times 10^{-6}$. In literature, BAN communication has been performed at $\mathrm{GHz}$ frequncy which is used for the ISM band. Thus, for simulation purposes, we use $1 \mathrm{GHz}$ as the transmission frequency. The transmitter is assumed to be a point source at $\boldsymbol{x}_{0}=\left(16 \mathrm{~cm}, \frac{\pi}{2}, 0\right)$. The receiver is assumed to be at a radial distance of $r_{0}=18 \mathrm{~cm}$ from the central cylindrical axis of the body. In the simulation, we move the receiver along the azimuth direction for varying values of $z_{0}$, i.e different heights from the transmitter.

\section{B. Implementation details}

For the simulation, we use (23) to calculate the 9 elements of the dyad in (22). Then, we substitute (22) in (20) to get the scattering DGF. However, (20) has an integration and an infinite length summation, and, as far as we are aware, explicit evaluation of this integral is not possible. Hence, we approximate it by a summation. We also truncate the infinite limits of both the integration and summation to finite numbers. It is evident from our simulations that these truncations are valid, as higher order vector eigen functions do not add 
significant energy to the resulting received electric field. Thus, we approximate (20) as

$$
\overline{\bar{G}}_{s}^{(22)}\left(\boldsymbol{x}, \boldsymbol{x}_{0}\right) \approx \sum_{\ell=-L}^{L} \sum_{n=-Q}^{Q} \frac{j}{8 \pi \eta_{2}^{2}} \overline{\bar{G}}_{n \Delta h \ell}^{(22)}\left(\boldsymbol{x}, \boldsymbol{x}_{0}\right) \Delta h
$$

where $L$ and $Q$ are appropriate truncation limits, and $\Delta h$ is the step size of the integration. For the parameters chosen in Section V-A, we notice that $N=40$ and $\Delta h \times L=60$, are appropriate values, as further increase in $N$ or $\Delta h \times L$ has no or very little effect on the results.

As the scattering DGF represents the EM propagation of the multipath reflection and transmission waves. It is more interesting to depict it's behaviour for the human body than the direct DGF component. Hence, we are only presenting the simulation plots for the electric field contributed from the scattering DGF.

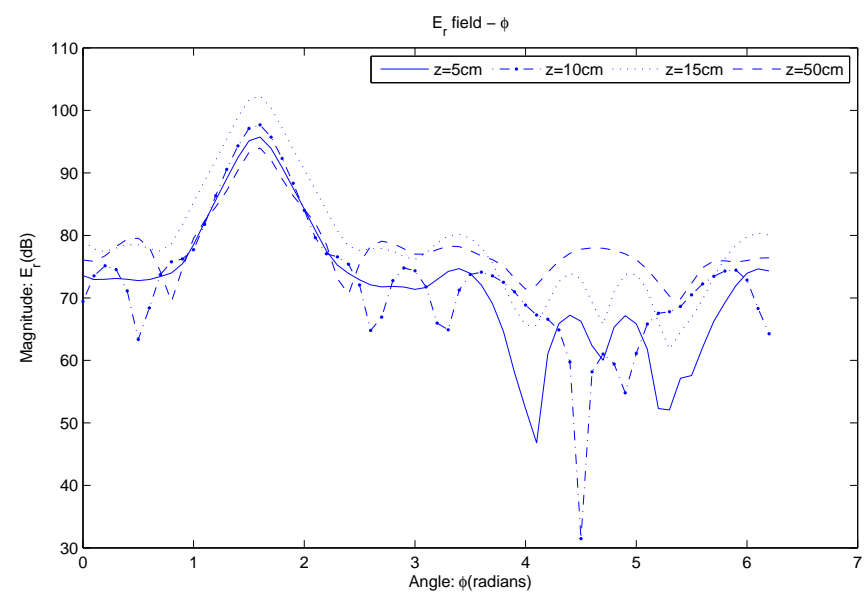

Fig. 1. Magnitude of scattered field component $E_{r}$ versus angle $\phi$ for different values of $z_{0}$. Radial distance of receiver is fixed at $r_{0}=18 \mathrm{~cm}$.

Using (30), we obtain three components of the electric field, one each in the $\widehat{\boldsymbol{r}}, \widehat{\phi}$ and $\widehat{z}$ directions. Each field component has been plotted as a function of azimuth $\phi \in[0,2 \pi)$. Each field component has also been plotted for different values of the receiver's $z$ coordinate $(z=5 \mathrm{~cm}, 10 \mathrm{~cm}, 15 \mathrm{~cm}, 50 \mathrm{~cm})$. The plots produced are shown in Figures 1,2 and 3. The total electric field, which's the vector addition of the three directional components is also plotted and presented in Figure 4.

In this paper, we do not wish to analyse these results. These simulations are simply used to illustrate the proposed model. As we mentioned earlier, this simple model is the first step in understanding the complex problem of wave propagation in and around the human body. We have not validated the model using any measurements nor any other numerical techniques. In near future, we expect to study (i) path loss and small scale fading characteristics around the simplified body model using the simulation technique proposed in this paper, and

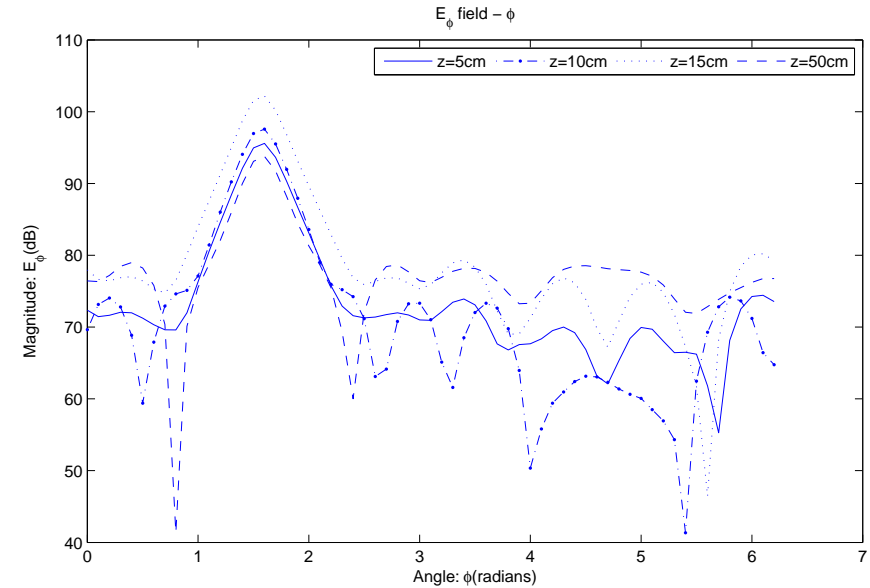

Fig. 2. Magnitude of scattered field component $E_{\phi}$ versus angle $\phi$ for different values of $z_{0}$. Radial distance of receiver is fixed at $r_{0}=18 \mathrm{~cm}$..

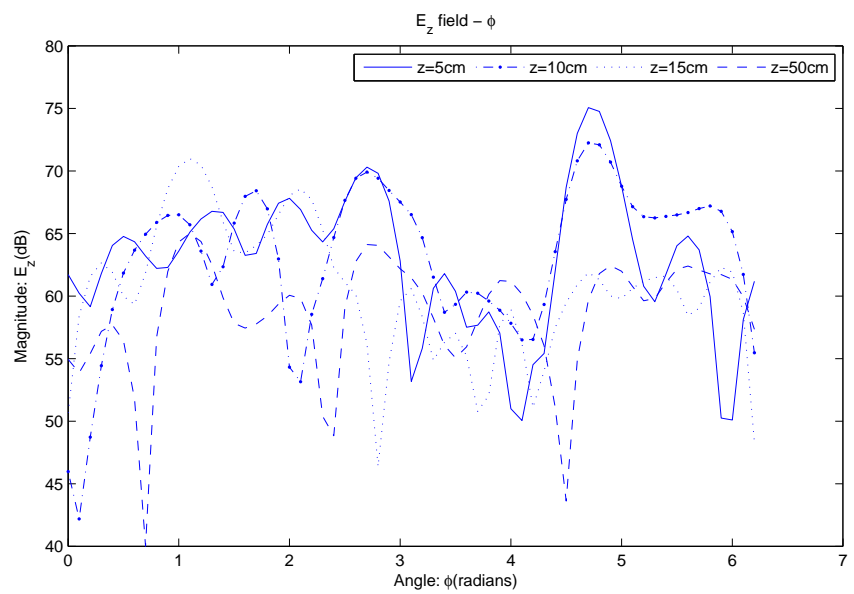

Fig. 3. Magnitude of scattered field component $E_{z}$ versus angle $\phi$ for different values of $z_{0}$. Radial distance of receiver is fixed at $r_{0}=18 \mathrm{~cm}$.

(ii) validate the model proposed in the paper by using FiniteDifference Time-domain (FDTD) method.

\section{CONCLUSions}

Using dyadic Green's functions we have derived an analytical channel model which describes the propagation characteristics of a cylindrical shaped human body. The model includes the cases where either the transmitter or receiver are inside or outside the body. Although, the exact expression includes an infinite integral and an infinite summation, simulations show that the integral can be limited to a finite range as well as approximated by a finite summation. The infinite sum can also be truncated to finite terms. These truncation limits seem to be related to the frequency of operation and the radius of the body. We expect to study details of these truncations as well as to prove the validity of the model against measurements and or numerical techniques such as FDTD method in our future work. 


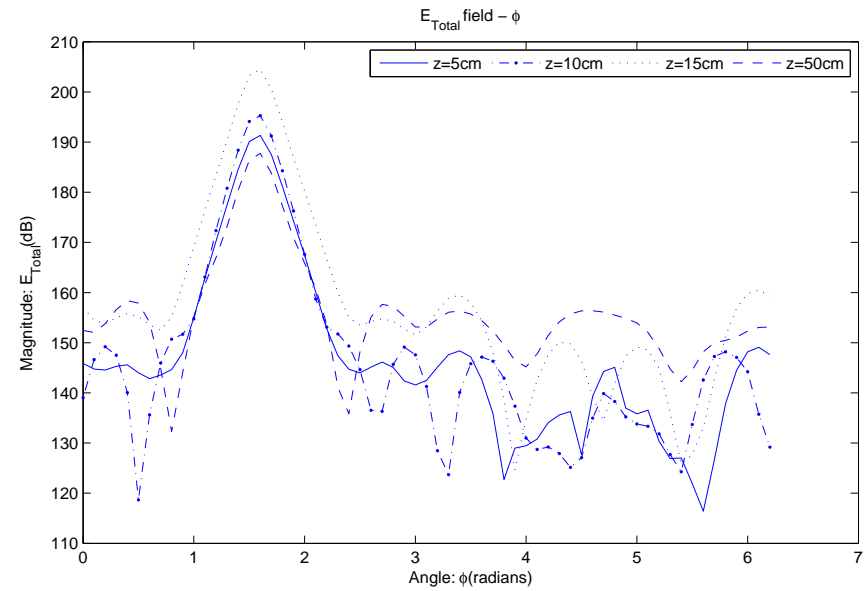

Fig. 4. Magnitude of total scattered field versus angle $\phi$ for different values of $z_{0}$. Radial distance of receiver is fixed at $r_{0}=18 \mathrm{~cm}$.

\section{REFERENCES}

[1] B. Gyselinckx, C.V.H Hoof, J. Ryckaert, R.F. Yazicioglu, P. Fiorini, and V. Leonov, "Human++: Autonomous wireless sensors for body area networks," Proc. Custom Integrated Circuits Conf, pp. 13-19, Sep 2005.

[2] F.E.H. Tay, M.N. Nyan, D.G. Guo, C.P. Ng, L. Xu, and C.T. Tan, "Memswear - biomonitoring system in a body area network," Proc. International Semiconductor Conference, vol. 1, pp. 207-214, Sep 2006.

[3] E. Lubrin, E. Lawrence, and K.F. Navarro, "Wireless remote healthcare monitoring with motes," Proc. Intl. Conf. on Mobile Business, pp. 235 241, July 2005.

[4] R. Bults, K. Wac, A.V. Halteren, D. Konstantas, V. Jones, and I. Widya, "Body area networks for ambulant patient monitoring over next generation public wireless networks," MobiHealth project, http://www.mobihealth.org), 2004.

[5] T. Zasowski, F. Althaus, M. Stager, A. Wittneben, and G. Troster, "Uwb for noninvasive wireless body area networks: Channel measurements and results," Proc. IEEE Conf. on Ultra Wideband Systems and Technologies, pp. 285-289, Nov 2003.

[6] A. Fort, J. Ryckaert, C. Desset, P.D. Doncker, P. Wambacq, and L.V. Biesen, "Ultra-wideband channel model for communication around the human body," IEEE Journal on Selected Areas in Communications, vol. 24, no. 4, pp. 927-933, April 2006.

[7] H. Ghannoum, C. Roblin, and X. Begaud, "Investigation of the uwb on-body propagation channel," http://uei.ensta.fr/roblin/papers/WPMC2006HGBANmodel.pdf, 2005.

[8] D. Nierynck, C. Williams, and A. Nix, M. Beach, "Channel characterisation for personal area networks," http://rose.bris.ac.uk/dspace/bitstream/1983/893/1/TD-05-115.pdf, Nov. 2007.

[9] A. Alomainy, Y. Hao, X. Hu, C.G. Parini, and P.S. Hall, "Uwb onbody radio propagation and system modelling for wireless body-centric networks," IEE Proc. Commun., vol. 153, no. 1, pp. 107-114, 2006.

[10] Y. Zhao, Y. Hao, A. Alomainy, and C. Parini, "Uwb on-body radio channel modelling using ray theory and sub-band fdtd method," IEEE Trans. On Microwave Theory and Techniques, Special Issue on UltraWideband, vol. 54, no. 4, pp. 1827-1835, 2006.

[11] J. Ryckaert, P.D. Doncker, R. Meys, A.D.L. Hoye, and S. Donnay, "Channel model for wireless communication around human body," Electronic Letters, vol. 40, no. 9, 2004.

[12] I.Z. Kovacs, G.F. Pedersen, P.C.F. Eggers, and K. Olesen, "Ultra wideband radio propagation in body area network scenarios," IEEE 8th Intl. symp. on Spread Spectrum Techniques and Applications, pp. 102-106, 2004.

[13] J.A. Ruiz, J. Xu, and S. Shiamamoto, "Propagation characteristics of intra-body communications for body area networks," 3rd IEEE Conf. on Consumer Communications and Networking, vol. 1, pp. 509-503, 2006.
[14] M.F Wong and J. Wiart, "Modelling of electromagnetic wave interactions with the human body," Journal - Comptes Rendus Physique, vol. 6, pp. 585-594, 2005.

[15] N. Siauve, R. Scorretti, N. Burais, L. Nicolas, and A. Nicolas, "Electromagnetic fields and human body: A new challenge for electromagnetic field computation," Int Journal for Computation and Maths. in Electrical and Electronic Eng., vol. 22, no. 3, pp. 457-469, 2003.

[16] K. Fuji, M. Takahashi, K. Ito, K. Hachisuka, Y. Terauchi, Y. Kishi, K. Sasaki, and K. Itao, "Study on the transmission mechanism for wearable device using the human body as a transmission channel," IEICE Proc. Intl. symp. on Antennas and Propagation, vol. E88-B, no. 6, pp. 2401-2410, 2005.

[17] M.A Stuchly and T.W. Dawson, "Interaction of low-frequency electric and magnetic fields with the human body," Proc. of IEEE, vol. 88, no. 5, pp. 643-664, 2000.

[18] T. Zasowski, G. Meyer, F. Althaus, and A. Wittneben, "Propagation effects in uwb body area networks," IEEE Intrenational Conference on $U W B$, pp. 16-21, 2005.

[19] Z. Xiang and Y. Lu, "Electromagnetic dyadic green's function in cylindrically multilayered media," IEEE Trans. on Microwave Theory and Techniques, vol. 44, no. 4, pp. 614-621, 1996.

[20] P.G. Cottis, G.E. Chatzarakis, and N.K. Uzunoglu, "Electromagnetic energy deposition inside a three-layer cylindrical human body model caused by near-field radiators," IEEE Trans. on Microwave Theory and Techniques, vol. 38, no. 8, pp. 415-436, 1990.

[21] S.M.S Reyhani and R.J. Glover, "Electromagnetic modeling of spherical head using dyadic green's function," IEE Journal, , no. 1999/043, pp. $8 / 1-8 / 5,1999$

[22] C.T. Tai, Dyadic Green's Functions in Electromagnetic Theory, IEEE, New York, 1993. 